\title{
Elderly Man With Weight Loss, Weakness, and Anorexia
}

Austin Hwang, MD

\section{Case Presentation}

An 81 year-old male who resides in Florida presents to the emergency department (ED) with complaints of weakness, 20 pound weight loss, and decreased appetite over the last six months. He is currently in the Philadelphia area to visit his son, and after his concerned son heard of these complaints, he brought his father to the ED. The patient indicates that he has become progressively weak over the last six months. He has also been increasingly fatigued, finding it difficult to walk even two blocks. A year ago, the patient walked two to three miles a day without a problem. Past medical history is significant for coronary artery disease, although he reports a normal stress test three months ago, stroke in 2004 with residual dysarthria, and melanoma on chest wall diagnosed in 2005 status-post resection. He lives with his wife and is a retired interior architect. He has not drunk alcohol in two years, has not smoked cigarettes in 20 years, and has never used illicit drugs. There is no history of cancer in his family, but significant history of stroke in his mother and sister. He saw his primary care physician for these symptoms in Florida, and a diagnosis had not been reached, but the patient indicates that he had a PET scan and a needle aspiration done on his right lung in the last month. He does not know the results of these tests.

On presentation, the patient was afebrile with a temperature of $97.0^{\circ}$ Fahrenheit, blood pressure of 164/77 $\mathrm{mm} \mathrm{Hg}$, heart rate of 57 beats/minute, and respiratory rate of 20 breaths/minute with pulse oximetry of $94 \%$ on room air. Physical exam was unremarkable, including the neurological exam. His cranial nerves, motor, sensory and cerebellar functions were all intact. Reflexes were $2+$ bilaterally in the upper and lower extremities, and patient had a normal gait.
This gentleman was admitted to the general floors, and given the patient's symptoms of weight loss, weakness, and decreased appetite, an occult malignancy seemed to be the most likely diagnosis. Chest radiograph on admission showed an ill-defined density in the right infrahilar region. PPD, HIV and ANA tests were negative, but ESR and CRP were elevated suggesting an inflammatory etiology. CT scan of the head, chest, abdomen, and pelvis was done on hospital day two. CT head without contrast showed no mass effect, bleed or intracranial lesions. CT chest, abdomen, and pelvis showed bilateral pleural effusions, ground glass opacities in both upper lobes, nonspecific scattered centrilobular nodules, consolidation/atelectasis in posterior segment of right lower lobe, three $0.5 \mathrm{~cm}$ nodules in the right lower lobe, and an infiltrative soft tissue mass surrounding both proximal collection systems in the retroperitoneal region.

On hospital day seven, PET scan results were obtained from Florida which showed right lung lower lobe and left lung lower lobe enhancement with greater attenuation found in the right basilar lung region. Right lung needle aspiration results from Florida obtained on hospital day nine were consistent with fibrotic tissue and no evidence of malignancy. The cardiothoracic surgeons were consulted to evaluate the patient for a VATS procedure in an attempt to obtain a tissue biopsy of the enhancement in the right lower lobe. MRI abdomen indicated that the soft tissue mass shown on CT abdomen and pelvis was consistent with retroperitoneal fibrosis and not malignancy. Despite the results of the needle aspiration from Florida, a repeat biopsy was scheduled via VATS on the right lower lobe of the lung. A right-sided chest tube was kept in place after the VATS, but was only necessary for one day. Pathology slides read two days later revealed pulmonary hyalinizing granulomas.
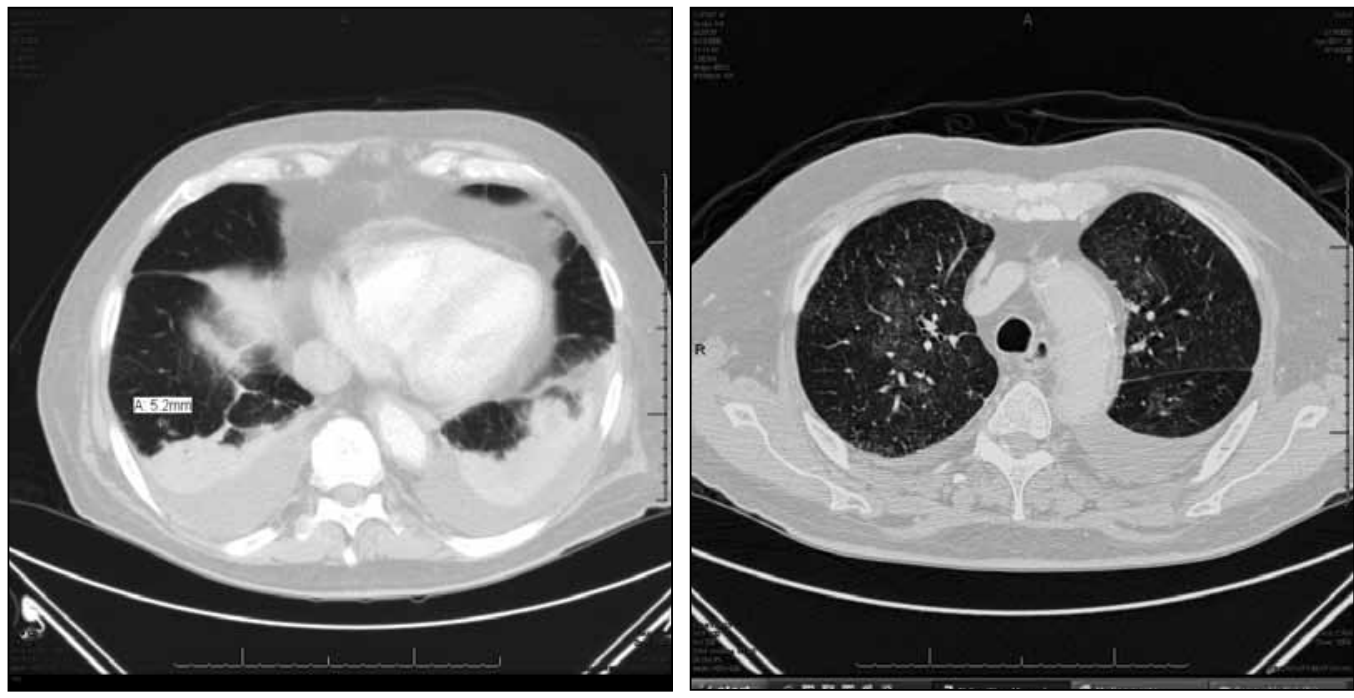

Figure 1. (left) CT chest demonstrates pathology in the right lower lung region and specifically, a $5.2 \mathrm{~mm}$ nodule.

Figure 2. (right)Bilateral ground glass opacities. 


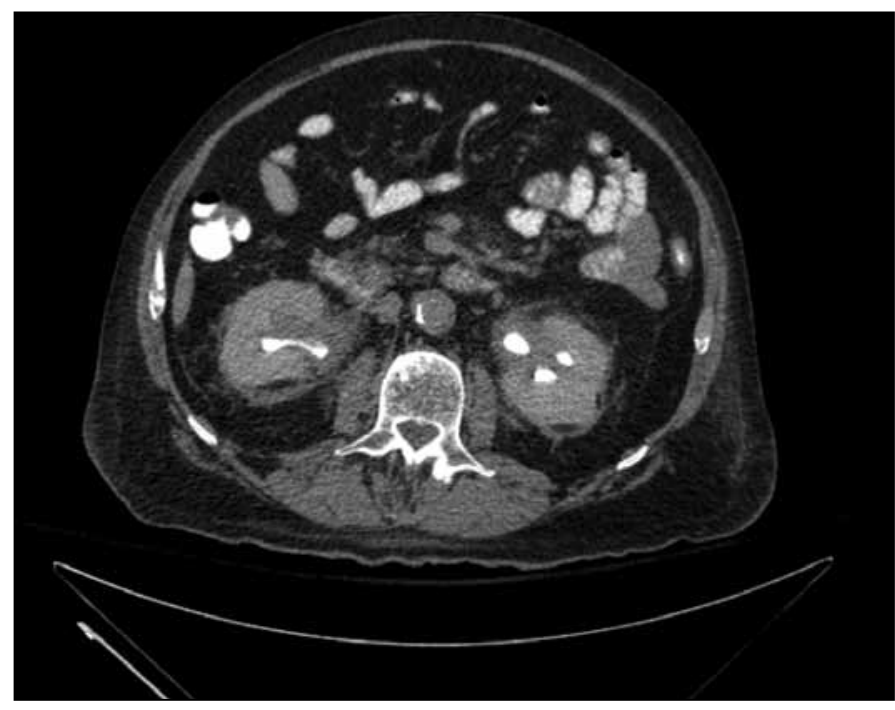

Figure 3. CT abdomen and pelvis with soft tissue infiltrates around bilateral kidneys

\section{Discussion}

Pulmonary hyalinizing granulomas were first described by Engleman et al in 1977. Just less than 100 cases have been reported since then. Symptoms include hemoptysis, pleuritic chest pain and cough. Retroperitoneal fibroses have been known to be associated with this condition. The disease is rare and idiopathic, but an autoimmune response is one of the leading theories to explain the etiology of this disease. Autoantibodies and circulating immune complexes have been detected in a few patients. The pulmonary lesions in these patients have been known to enlarge or regress spontaneously, and a search for an inciting factor for these events has so far been fruitless. In Thorax, an article by Dent et al in 1983 discussed a patient with a diagnosis of pulmonary hyalinizing granulomas and retroperi-

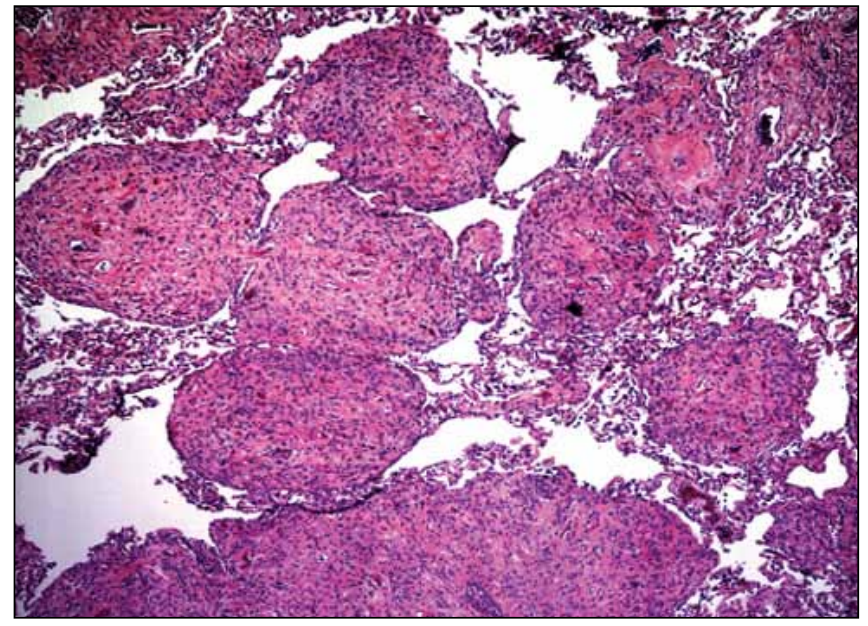

Figure 5. Right lower lobe pulmonary biopsy evinces pulmonary hyalinizing granulomas.

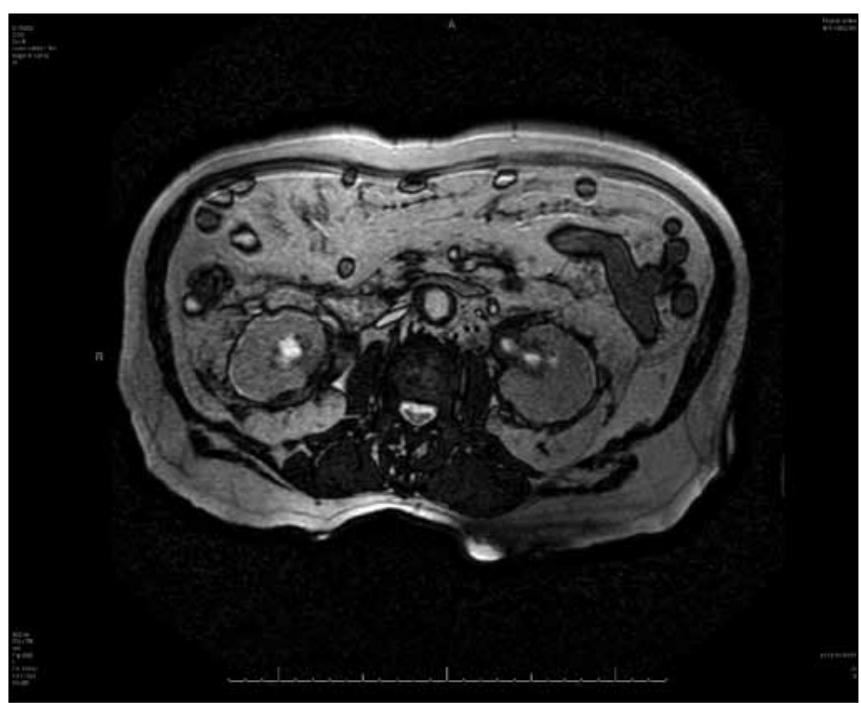

Figure 4. MRI abdomen with soft infiltrates around bilateral kidneys.

toneal fibrosis who worked in the oil industry for many years and had copious exposure to toxic fumes. There was some thought that oil could be an instigator of these spontaneous growths of the granulomas, but the histology did not support this theory as there were not many mineral oil droplets present on the slides. A typical histologic slide consistent with pulmonary hyalinizing granulomas shows densely matted, stout, hypocellular collagen fibers with fine granular stippling with sporadic calcium deposits. The presence of amyloid fibers can co-occur with these granulomas also.

The biopsy results for our 81 year-old male were consistent with pulmonary hyalinizing granulomas. Initially, there were plans to biopsy the retroperitoneal fibrosis after the patient's VATS, but given previous case reports describing a strong correlation between retroperitoneal fibroses and our primary diagnosis, the biopsy never occurred. This patient was started on high dose prednisone at $80 \mathrm{mg}$ by mouth once a day. He was to be tapered off of the prednisone slowly. After a few days of treatment, the patient's weakness and appetite improved. The patient received physical therapy for a couple more days and was discharged from the hospital. The patient is to follow up closely with his primary care physician in Florida.

\section{References}

1. Engleman P, Liebow AA, Gmelich J, Friedman PJ. Pulmonary hyalinising granuloma. Am Rev Respir Dis 1977; 115: 997-1008.

2. Hawk WA, Hazard JB. Sclerosing retroperitonitis and sclerosing mediastinitis. Am J Clin Pathol 1959; 32: 321-334.

3. Hammar SP, Gortner D, Stanford S, Bockus D. Lymphomatoid granulomatosis: association with retroperitoneal fibrosis and evidence of impaired cell-mediated immunity. Am Rev Respir Dis 1977; 115: 1045-50.

4. Dent RG, Godden DJ, Stovin PGI, Stark JE. Pulmonary hyalinising granuloma in association with retroperitoneal fibrosis. Thorax 1983; 38: 955-956. 